http://jmscr.igmpublication.org/home/

ISSN (e)-2347-176x ISSN (p) 2455-0450

crossref DOI: https://dx.doi.org/10.18535/jmscr/v7i11.155

Journal Of Medical Science And Clinical Research

IGM Publication

An official Publication of IGM Publication

Original Article

\title{
Cultural adaptation, reliability, and validation of the Mizo-Version of Beck Depression Inventory (BDI) in the rural Northeast Indian population
}

\author{
Authors \\ Andrew Lalchhuanawma', Divya Sanghi ${ }^{2 *}$ \\ Department of Physiotherapy, Faculty of Applied Science, \\ Manav Rachna International Institute of Research \& Studies, Faridabad, India \\ *Corresponding Author \\ Divya Sanghi Ph.D \\ Associate Professor, Faculty of Applied Sciences, \\ Manav Rachna International Institute of Research \& Studies, Faridabad, India
}

\begin{abstract}
Introduction: The Beck Depression Inventory (BDI) is a popular instrument which is used extensively for measuring the severity of depression, for both screening purposes and in clinical practice. The BDI consists of 21 items; each item was scored on a 4-pointer Likert scale starting from 0 to 3, giving the total possible items score to 63.Many of the existing patient-reported outcome measures were designed and established for the Western and European English speaking nation. The need for the cross-cultural adaptation and linguistic translation arose as the psychometric properties of BDI scale has never been validated in Mizo culture of the rural northeast Indian population.

Objective: To establish the reliability and construct validity of the Mizo-BDI. This study aims to culturally adapt and translate the patient reported outcome measure of BDI into Mizo tawng (an official language of Mizoram).

Method: 49 community dwelling citizens from the rural northeast Indian primary health-care settings participated for the study. Subjects participated voluntarily in the study after taking a formal consent. The study followed the recommended guidelines for back-translation of the English-BDI into Mizo-BDI.

Results: Internal consistency determined by Cronbach alpha was found to have a good reliability $(\alpha=0.89)$, test-retest reliability determined by Intraclass correlation coefficient (ICC) showed an excellent reliability (ICC =0.96, 95\% CI=0.94-0.98). For construct validity test, Pearson's correlation coefficient analyzed the relationship between Numerical Pain Rating Scale (NPRS) and BDI and found to have a slight relationship and significant $(r=0.31, p<0.05)$

Conclusion: The study results validated the M-BDI to be easy to comprehend, reliable and valid assessment tool for measuring the severity of depression in the Mizo speaking rural population of Mizoram, India.

Keywords: depression, reliability, validity, beck depression inventory.
\end{abstract}

\section{Background}

Depression is a common mental disorder that slowly becomes a global phenomenon. In this day and age, depression is becoming more pandemic reaching the far corners of the globe. The rate at which it affects a significant number of 
individuals is at an alarming high. According to the statistics released in 2015 by the World Health Organization (WHO), a sharp increase in the incidence rate of $18.4 \%$ was observed between the years 2005 to 2015 . It is further estimated that depression accounts for over 322 million people, which is roughly $4 \%$ of the world's population, and women were found to be more depressed than men. ${ }^{[1][2][3]}$ WHO has predicted depression to be among the leading cause of disease burden worldwide by the year 2020. ${ }^{[3]}$

The state of an individual's mental health status has become a focal point of interest. Depression isa serious mental health issues which is associated with suicide ideation and other cause. With an early diagnosis and timely help, the risk of chronic depressive symptoms and other negative consequences could be avoided. ${ }^{[4]}$

The Beck Depression Inventory (BDI) is a popular instrument which is used extensively for measuring the severity of depression.BDI is a selfreported scale which is used forboth screening purposes and in clinical practice. ${ }^{[4]}$ Although there are several patient-reported outcome measures (PROM) for evaluating the severity of depression, the BDI was selected as it is the most widely accepted and convenient tool available. The other relevant available outcome measures includes; Beck Anxiety Inventory (BAI), Beck Hopelessness Scale (BHS) and the Suicide Ideation Scale (SIS). ${ }^{[5]}$

The BDI was originally developed in English in the year 1961, since then it came across various adaptations and translations into many languages with high degree of reliability and validity. Major changes occurred twice within a substantial period of time; first, in 1978 with a revised format as the BDI- IA, and later in 1996 after undergoing subsequent changes as the BDI- II. ${ }^{[4][6]}$

This study accentuated the standard protocol for selecting an appropriate patient-reported outcome measure, which depends upon the various properties such as; the measured content and the feasibility of psychometric properties, the reliability and validity of the construct measured, quantifiable and sustenance of scores, and the quality of adaptation and translation. ${ }^{[7]}$

Of the many existing patient-reported outcome measures available, almost all the instruments were designed and established for the Western and European English speaking nation. ${ }^{[5]}$ Crosscultural adaptation and linguistic translation is required for standardization of the specific instrument. The psychometric properties of BDI scale has never been validated in Mizo language (official language of Mizoram) of the northeast Indian population.

The English version of the BDI is proven to be a reliable tool for measuring the severity of depression among the Asian population, it has never been validated among the northeast Indian population. At present, no self-reported instruments have ever been validated and culturally adapted for the Mizo speaking population. This study will provide an appropriate result for the culturally oriented respondents in evaluating the severity of depression even among the rural population of Mizoram state, India where English is not spoken as means of communication. The aim of the study is to establish the reliability and construct validity of the Mizo version of the BDI in clinical setting. The objective is to standardize the self-reported outcome measure, for cultural adaptation and translation of the BDI into Mizo version.

\section{Materials and Methods Method}

A sample for the study was taken from the outpatient department of the rural primary health centre, Lunglei district, Mizoram. A total of 49 subjects participated voluntarily for the study. Participants were handed the self-reported questionnaire of the BDI-Mizo version and told to give the survey, and data was collected. The socio-demographic data and the anthropometric data were also obtained from each participant. Subjects were again re-assessed after 1 week with the same self-reported parameters of the BDI- 
Mizo version but with inter-changing the itemized order.

This study was approved by the Institutional Ethical Review Board, Manav Rachna International Institute of Research and Studies, Faridabad. The present study proceeded in accordance with the Belmont code of ethical principles, and a formal written consent was taken from each participant. Each subject was briefed about the basic research ethics which hangs ont he five pillars i.e. informed consent, confidentiality, anonymity, beneficence and privacy. ${ }^{[8]}$

\section{Instruments}

\section{Beck Depression Inventory (BDI)}

The BDI, developed by Beck et al. is a selfinventory questionnaire consisting of 21 items. The main objective of the BDI test is to measure the severity of depression for the past couple of weeks along with the current state of depressive symptoms. Each item is scored on a 4-pointer Likert scale starting from 0 to 3, giving the total possible items score to 63 . The resulting scores from the items provide the severity of depressive symptoms; 0-9 is normal, between 10 and 16 is minimal, between 17 and 20 is borderline, 21 to 30 is moderate, 31 to 40 is severe, and above 40 is extreme depression. Study subjects after confirming their participation will be asked to tick or circle the one choice from the given sub-items that best describe their current emotional state. ${ }^{[9]}$

\section{Numerical Pain Rating Scale (NPRS)}

The NPRS scale is the most widely used selfreported instrument for measuring the intensity of pain. It consists of $11 \mathrm{sub}$ points on a Likert Scale, measuring from $0-10$. A score of 0 denotes no pain, whereas a maximum score of 10 signifies the worst type of pain imaginable. Subjects are instructed to circle/ mark on the 11 pointer Likert Scale that best describes their pain. NPRS is a common evaluation scale, used for measuring the intensity of pain. The scale consists of 11 sub points starting from 0 to 10 . ' $\mathrm{O}$ ' denotes normal without pain whereas, 10 will denote the excruciating worst type of pain. After instructing each subject, all the study subjects will be asked to rate the levels of pain. ${ }^{[10]}$

\section{Procedure}

\section{Guidelines for Cross-Cultural Adaptation}

A recent journal report doesn't support the formation or development of new questionnaires, rather stating the need togather more information on the ones already standardized and validated in the English language. This study followed a standard protocol for translation and cultural adaptation of BDI into Mizo language. Beaton et $\boldsymbol{a l} .{ }^{[11]}$ developed the recommended guidelines for any linguistic translation and cross-cultural adaptation. It comprised of 6 processes which is shown in Figure 1.

Process 1: The English version of the self-reported outcome measure of BDI was translated into Mizo language by two assessors or translators.

Process 2: The two translated variables were reviewed by an external expert committee, following which it was adapted and compiled into a single Mizo variable.

Process 3: Two independent natives of Mizoram speaking impeccable English were asked for a backward translation of the adapted Mizo BDI into English. Both were blinded to the original NDI English version.

Process 4: The two versions of the backward language translations were submitted to the experts committee. The panel board comprised of health professionals, expert in linguistic translations and scientific researchers.

Process 5: The pre-final version of the BDI-Mizo was prepared by the expert committee. This tool underwent a trial and error sample testing for determination of face validity.

Process 6: Submission of all the reports to the committee is the last step in the adaptation process. The expert panel reached equivalence on the essential four broad areas as described by Beaton and his associates; semantic equivalence (ensure equivalent word-by-word translation), idiomatic equivalence (ensuring similar colloquial 
expressions), experiential equivalence (making sure that the experiential activities of daily living capture the intended cultures), and conceptual equivalence (making sure the item stays on course with the main concept)

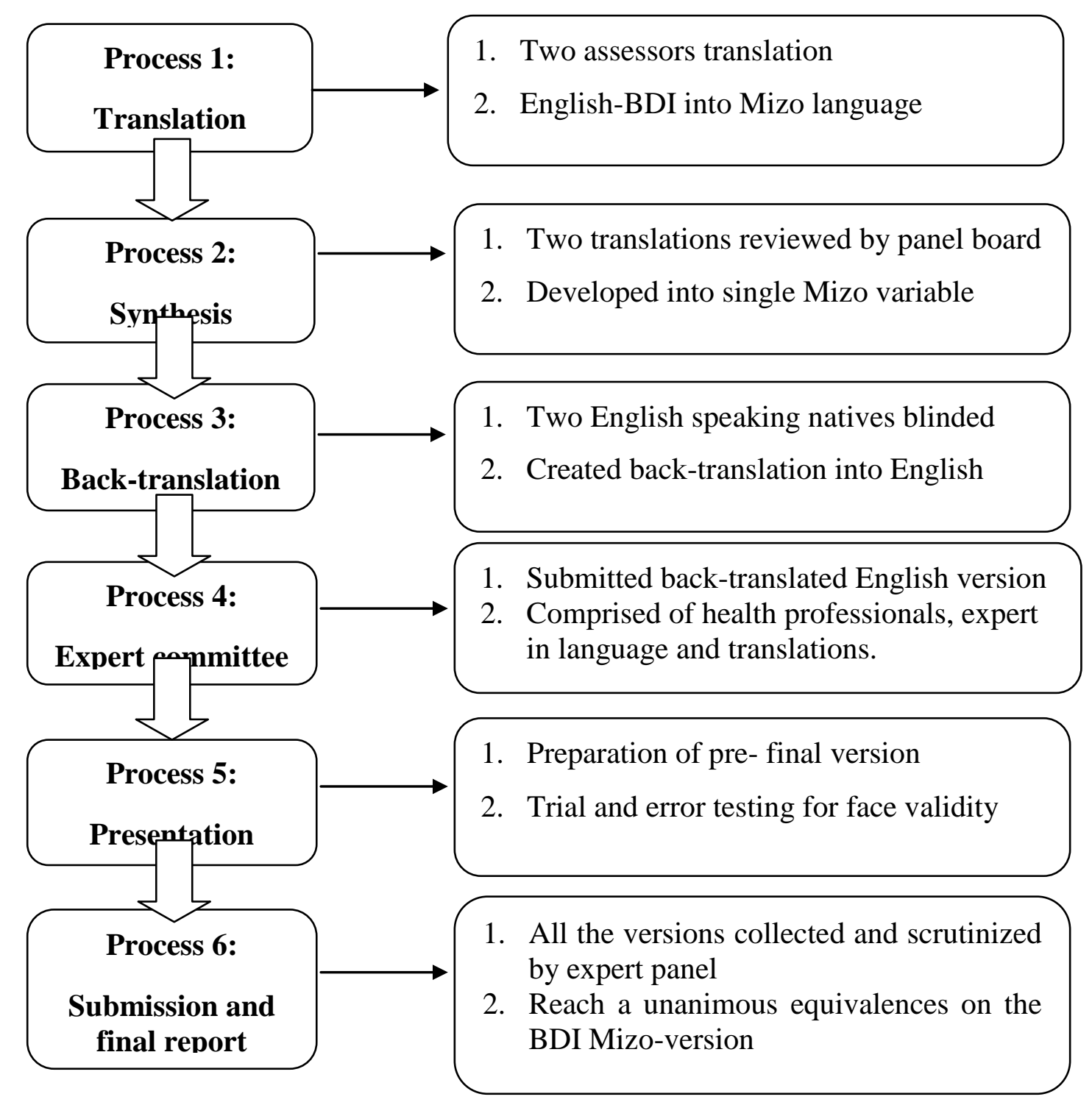

Figure 1: Processes of Linguistic Translation and Cultural Adaptation.

\section{Data Analysis}

\section{Validity testing of Psychometric properties}

Data for all the variables of the study samples were analyzed using IBM SPSS software packages version 24.0. Reliability testing for internal consistency was determined by using Cronbach's alpha. The test-retest reliability was determined by using the Intraclass correlation coefficient (ICC). For determination of construct validity, the relationship between the items scored on Numerical Pain Rating Scale (NPRS) and BDI was analyzed using the Pearson's correlation coefficient.

\section{Results}

This study consisted of 49 individuals, in which 17 were males and 32 females. The mean age was 34.96 with age group between 19 to 55 . The demographic data and descriptive statistics are presented in table 1 and 2. 


\begin{tabular}{|l|c|c|c|}
\hline \multicolumn{3}{|l|}{ Table 1. Demographic Data } \\
\hline Variables & & Mean & SD \\
\hline Age (yrs) & $\begin{array}{c}\text { Minimum- } \\
19\end{array}$ & 34.96 & 9.43 \\
& $\begin{array}{c} \\
\end{array}$ & $\begin{array}{c}\text { Maximum- } \\
55\end{array}$ & \\
\hline & & Frequency & Percentage \\
\hline Gender & Male & 17 & 34.7 \\
\cline { 2 - 4 } & Female & 32 & 65.3 \\
\hline
\end{tabular}

\begin{tabular}{|l|c|c|}
\hline \multicolumn{3}{|c|}{ Table 2. Descriptive Statistics } \\
\hline Variables & Mean & SD \\
\hline NPRS & 4.59 & 2.12 \\
\hline BDI & 14.92 & 8.93 \\
\hline
\end{tabular}

\section{Reliability}

For establishing the internal consistency of MBDI, the Cronbach's alpha estimates was found to have a good reliability with $\alpha=0.89$. All the total item scores for the samples were positively correlated and statistically significant $(\mathrm{p}<0.01)$. The calculated alpha value if item deleted ranges between 0.882 to 0.890 which means each of the items contributed significantly for the M-BDI score.Table 3 represents the M-BDI for means, standard deviation, item total correlations and alpha if item deleted.

\begin{tabular}{|c|c|c|c|c|}
\hline Items & Mean & SD & $\begin{array}{l}\text { Corrected Item- } \\
\text { Total Correlation }\end{array}$ & $\begin{array}{l}\text { Cronbach's Alpha if } \\
\text { Item Deleted }\end{array}$ \\
\hline Sadness & 0.79 & 0.76 & 0.56 & 0.884 \\
\hline Pessimism & 0.71 & 0.84 & 0.53 & 0.884 \\
\hline Past failure & 0.77 & 0.84 & 0.53 & 0.885 \\
\hline Loss of pleasure & 0.63 & 0.69 & 0.46 & 0.886 \\
\hline Guilty feelings & 1.24 & 0.72 & 0.38 & 0.889 \\
\hline Punishment feelings & 0.59 & 0.76 & 0.50 & 0.885 \\
\hline Self-dislike & 0.55 & 0.79 & 0.52 & 0.885 \\
\hline Self-criticalness & 0.69 & 0.79 & 0.50 & 0.885 \\
\hline Suicidal thought & 0.28 & 0.54 & 0.34 & 0.889 \\
\hline Crying & 0.79 & 0.88 & 0.54 & 0.884 \\
\hline Agitation & 0.73 & 0.72 & 0.51 & 0.885 \\
\hline Loss of interest & 0.61 & 0.86 & 0.61 & 0.882 \\
\hline Indecisiveness & 0.79 & 0.64 & 0.50 & 0.886 \\
\hline Worthlessness & 0.63 & 0.66 & 0.30 & 0.890 \\
\hline Loss of energy & 0.79 & 0.76 & 0.57 & 0.883 \\
\hline Sleep changes & 0.75 & 0.80 & 0.54 & 0.884 \\
\hline Irritability & 0.91 & 0.78 & 0.53 & 0.884 \\
\hline Appetite changes & 0.57 & 0.81 & 0.49 & 0.886 \\
\hline Concentration & 0.59 & 0.70 & 0.49 & 0.886 \\
\hline Tiredness & 0.87 & 0.66 & 0.32 & 0.890 \\
\hline Loss of sex interest & 0.55 & 0.76 & 0.57 & 0.883 \\
\hline
\end{tabular}

The test-retest intra-rater reliability using intraclass correlation coefficient (ICC), two way mixed effects model, absolute agreement definition was found to have an excellent reliability with $\mathrm{ICC}=0.96,95 \%$ confidence interval $(0.94-0.98)$. Table 4 represents the Intraclass correlation coefficient including the 95\% Confidence Interval (CI) with a significant value.

\begin{tabular}{|l|l|c|c|c|c|c|c|}
\hline Table 4: Intraclass Correlation Coefficient \\
\hline & Intraclass & $\mathbf{9 5 \%}$ Confidence Interval & \multicolumn{3}{|c|}{ F Test with True Value 0 } \\
\cline { 3 - 8 } & Correlation & Lower Bound & Upper Bound & Value & df1 & df2 & Sig \\
\hline Single Measures & 0.96 & 0.94 & 0.98 & 58.924 & 48 & 48 & .000 \\
\hline Average Measures & 0.98 & 0.97 & 0.99 & 58.924 & 48 & 48 & .000 \\
\hline Two-way mixed effects model where people effects are random and measures effects are fixed. \\
\hline
\end{tabular}




\section{Validity}

The Pearson's Correlation Coefficient analyzed the $p$ value and indicates a slight relationship $(\mathrm{r}=0.31)$ and statistically significant correlation $(\mathrm{p}<0.05)$ between NPRS and M-BDI. The correlation between NPRS and BDI with significant level at 0.05 is given in table 5 , and their correlation is represented by a simple scatter graph (figure 2).

\begin{tabular}{|l|c|c|c|}
\hline \multicolumn{4}{|c|}{ Table 5. Correlations between NPRS and BDI } \\
\hline \multirow{4}{*}{ NPRS } & $\begin{array}{c}\text { Pearson } \\
\text { Correlation }\end{array}$ & 1 & $0.31^{*}$ \\
\cline { 2 - 4 } & Sig. (2-tailed) & & .028 \\
\cline { 2 - 4 } & $\mathrm{N}$ & 49 & 49 \\
\hline \multirow{4}{*}{ BDI } & $\begin{array}{c}\text { Pearson } \\
\text { Correlation }\end{array}$ & $0.31^{*}$ & 1 \\
\cline { 2 - 4 } & Sig. (2-tailed) & .028 & \\
\cline { 2 - 4 } & $\mathrm{N}$ & 49 & 49 \\
\hline $\begin{array}{l}\text { *Correlation } \\
\text { tailed). }\end{array}$ & is significant at the 0.05 level (2- \\
\hline
\end{tabular}

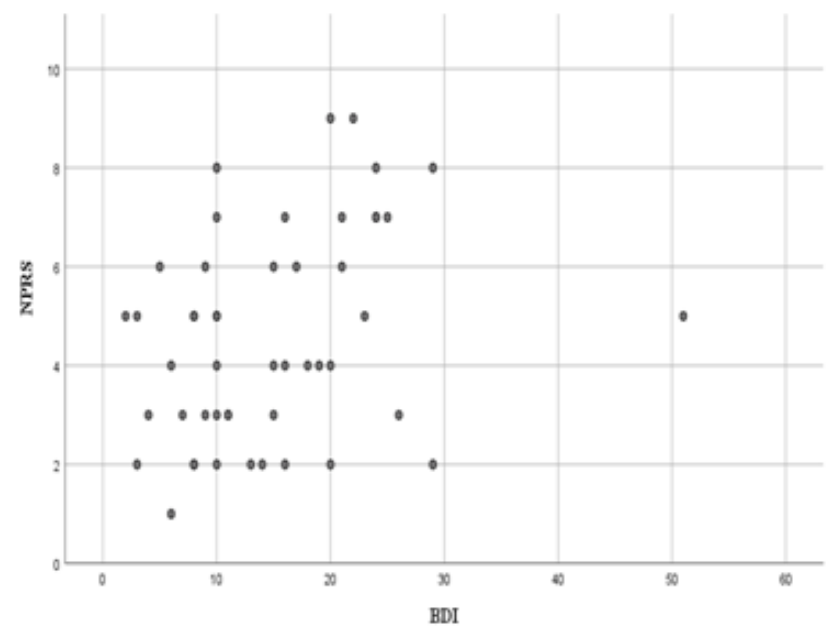

Figure 2 Simple scatter of NPRS by BDI

\section{Discussion}

The present study aims to provide cultural adaptation and linguistic translation with the objectives of determining the reliability and validity of the Mizo version of the BDI. Samples for the study consisted of individuals who visited the out-patient department with complaints of physical pain associated symptoms. The selected candidates were asked to rate the level of their pain using the NPRS variable which is a 11 pointer scale. A total of 49 subjects (17 males and
32 females) participated for the study, with the mean age of $34.96 \pm 9.43$ (between 19-55 years). The mean score for the variables NPRS and BDI was $4.59 \pm 2.12$ and $14.92 \pm 8.93$ respectively. Low mean scores for depression on the BDI scale couldbe explained by selection criteria which did not exclusively include depressive patients.

Biasing from an extraneous variable which could influence the result was ruled out as the M-BDI has no ceiling/ floor effects as the maximum and minimum possible scores from respondents did not exceed the $15 \%$ barrier. No patients were reported to have achieved the maximum and minimum possible scores.

Reliability testing includes internal consistency and test-retest reliability. The Cronbach's alpha is a common method that measures the internal consistency, which is the interrelationship between each items score as well as the individual item score with the total item score. The value of Cronbach's alpha $>70$ signifies a good correlation whereas a low value implies no relationship between the items. The alpha value $(\alpha=0.89)$ of M-BDI for the study indicated an excellent internal reliability, and the score was consistent with that of previous research findings among the Korean population. ${ }^{[4]}$

The intraclass correlation coefficient (ICC) index is extensively used for test-retest reliability analysis and, intra-rater and interrater reliability analysis. ${ }^{[12]}$ ICC values $<0.5,0.5$ to $0.75,0.75$ to 90 , and $>90$ indicates poor, moderate, good, and excellent reliability, respectively. The ICC value of the M-BDI after 7 days interval was $0.96,95 \%$ CI $(0.94-0.98)$ suggestive of an excellent reliability and a significant correlation with the total BDI score. Based upon the observed findings on the internal consistency and test-retest reliability, the study indicates the M-BDI to be reliable, consistent, easily adaptable and valid assessment tool for the Mizo culture.

Construct validity is a type of test, designed to measure what the construct item actually claimed to measure. Tests construct that claims to have a correlation, indeed, related are called Convergent 
validity. On the other hand, test construct that doesn't claim to have a relation, in fact, unrelated are Divergent validity. ${ }^{[13]}$ The Pearson's Correlation Coefficient ' $r$ ' is used to measure the construct validity, where ' $r$ ' ranges from +1 to -1 . The closer the value is to -ve or +ve, the stronger is the relationship, whereas the value 0 indicates no relationship between the variables. The correlation between NPRS and M-BDI was assessed by Pearson's analysis and found to have a slight relationship $(\mathrm{r}=0.31)$ and a statistically significant correlation $(\mathrm{p}<0.05)$. Previous studies found depression to be associated with physical symptoms of pain which correlated with the findings in this study. ${ }^{[14,15]}$

\section{Conclusion}

The Mizo version of the BDI is found to be reliable, easy to comprehend, consistent and valid instrument tool for assessing severity of depressive symptoms in the Mizo speaking rural population of northeast India. It can be recommended for the Mizo culture in clinical practice and research settings.

\section{Competing interests}

The authors declared no competing interest to exist.

\section{Funding or grants}

The authors gained no financial benefit and no funding was received for the study.

\section{References}

1. World Health Organization (WHO). 2015, Depression https:// www.who.int/ mental health/ management/depression/en/

2. Erica Orange and Jared Weiner https:// medium.com/ positive-returns/ a-mentalhealth-pandemic-is-the-world-gettingmore-addicted-anxious-and-lonely4e45bf533ae5

3. Tucci, V., \& Moukaddam, N. (2017). We are the hollow men: The worldwide epidemic of mental illness, psychiatric and behavioral emergencies, and its impact on patients and providers. Journal of emergencies, trauma, and shock, 10(1), 46.

4. Lee, E. H., Lee, S. J., Hwang, S. T., Hong, S. H., \& Kim, J. H. (2017). Reliability and Validity of the Beck Depression InventoryII among Korean Adolescents. Psychiatry investigation, 14(1), 30-36.

5. Ozcelik, H. S., Ozdel, K., Bulut, S. D., \&Orsel, S. (2015). The reliability and validity of the Turkish version of the Beck Scale for Suicide Ideation (Turkish BSSI). Klinik Psikofarmakoloji Bülteni / Bulletin of Clinical Psychopharmacology, 25(2), 141-150.

6. Beck AT, Steer RA, Brown GK. BDI-II: Beck Depression Inventory Manual. 2nd Ed. San Antonio, TX: Psychological Corporation; 1996.

7. Reeve BB, Wyrwich $\mathrm{KW}$, Wu AW, Velikova G, Terwee GB, et al. ISOQOL recommends minimum standards for patient-reported outcome measures used in patient-centered outcomes and comparative effectiveness research.

8. Barrow JM, Khandhar PB. Research Ethics. [Updated 2019 Jun 22]. In: Stat Pearls [Internet]. Treasure Island (FL): Stat Pearls Publishing; 2019 Jan-. Available from:

https://www.ncbi.nlm.nih.gov/books/NBK 459281/

9. Beck AT, Ward CH, Mendelson M, Mock J, Erbaugh J. An inventory for measuring depression. Arch Gen Psychiatry 1961;4(6):561-71.

10. Erin EK, Timothy SC and Weinberger M. Accuracy of the Pain Numeric Rating Scale as a Screening Test in Primary Care. Society of General Internal Medicine. (2007).

11. Beaton DE, Bombardier C, Guillemin F, et al. Guidelines for the process of crosscultural adaptation of self-report measures. Spine 2000; $25: 3186$ - 91. 
12. Terry K. Koo, Mae Y. Li. A Guideline of Selecting and Reporting Intraclass Correlation Coefficients for Reliability Research. Journal of Chiropractic Medicine (2016) 15, 155-163.

13. Cronbach LJ, Meehl PE. Construct validity in psychological tests. Psychol Bull. 1955 Jul;52(4):281-302.

14. Madhukar H. Trivedi. The link between Depression and Physical Symptoms.The Primary Care Companion to the Journal of Clinical Psychiatry, 2004; 6(suppl 1): 1216.

15. Ohayon MM, Schatzberg AF. Using pain to predict depressive morbidity in the general population. Archives of General Psychiatry. 2003; 60: 39-47. 